\title{
Fundamental Nanoscale Phenomena and Processes
}

National Cancer Institute

\section{Source}

National Cancer Institute. Fundamental Nanoscale Phenomena and Processes. NCI

Thesaurus. Code C53670.

Discovery and development of fundamental knowledge pertaining to new phenomena in the physical, biological, and engineering sciences that occur at the nanoscale. Elucidation of scientific and engineering principles related to nanoscale structures, processes, and mechanisms. 